\title{
A Case of Vertebral Artery Fusiform Aneurysm Treated by Flow Alteration: Successful Prediction of Therapeutic Effects Using Computational Fluid Dynamics
}

\author{
Yoichi Miura, ${ }^{1}$ Fujimaro Ishida, ${ }^{2}$ Yusuke Kamei, ${ }^{3}$ Masanori Tsuji, ${ }^{2}$ Masato Shiba,${ }^{4}$ Hiroshi Tanemura, ${ }^{2}$ \\ Yasuyuki Umeda, ${ }^{3}$ Shinichi Shimosaka, ${ }^{2}$ and Hidenori Suzuki ${ }^{4}$
}

The treatment of intracranial complicated aneurysms remains challenging. In patients with complicated aneurysms that are neither clippable nor coilable, flow alteration treatment (FAT) with a combined procedure of proximal/distal occlusion or trapping of an aneurysm with bypass surgery has been reported. However, it is difficult to predict whatever FAT can achieve aneurysmal obliteration without ischemic complications. A 69-year-old female was incidentally diagnosed with a left vertebral artery (VA) fusiform aneurysm distal to the left posterior inferior cerebellar artery (PICA). Because one-year follow-up threedimensional computed tomography angiography showed that the aneurysm grew significantly, surgical management was considered the therapy of choice. For determining treatment strategies, we assumed left VA occlusion at the proximal to the left PICA as a FAT model and performed computational fluid dynamics (CFD) analyses. The FAT model had much lower wall shear stress and shear rate at the aneurysm dome than presumed thresholds necessary to thrombus formation, while those at the PICA were obviously higher than the thresholds, and streamlines into the left PICA from the distal VA were preserved. These findings theoretically meant that surgical occlusion of the left VA proximal to the left PICA and aneurysm would induce intra-aneurysmal thrombus formation with preservation of the left PICA flow. The treatment was performed successfully and achieved the predicted results. CFD simulations may be useful to predict effects of FAT for complicated aneurysms.

Keywords: cerebral aneurysm, computational fluid dynamics, flow alteration treatment, thrombus formation

\footnotetext{
${ }^{1}$ Department of Neurosurgery, Suzuka Kaisei Hospital, Suzuka, Mie, Japan

${ }^{2}$ Department of Neurosurgery, Mie Chuo Medical Center, National Hospital Organization, Tsu, Mie, Japan

${ }^{3}$ Department of Neurosurgery, Mie Prefectural General Medical Center, Yokkaichi, Mie, Japan

${ }^{4}$ Department of Neurosurgery, Mie University Graduate School of Medicine, Tsu, Mie, Japan
}

Received: January 30, 2017; Accepted: March 27, 2017

Online August 23, 2017

Copyright $\subset 2017$ by The Japan Neurosurgical Society This work is licensed under a Creative Commons AttributionNonCommercial-NoDerivatives International License.

\section{Introduction}

In spite of advances in surgical clipping and endovascular coiling, some complicated aneurysms remain impossible to be treated using the conventional methods. For such a case, flow alteration treatment (FAT) has been suggested. ${ }^{1)}$

Wall shear stress (WSS) and shear rate were identified in the 1970s and subsequently investigated for their potential impact on thrombus formation. ${ }^{2,3)}$ Thrombus formation is induced in a condition of flow stagnation. Corbett et al. ${ }^{4)}$ conducted the experimental study to determine the threshold values of WSS and shear rate that are necessary to thrombus formation in a condition of flow stagnation. They revealed a WSS threshold of $0.41 \mathrm{~Pa}$ and a shear rate threshold of 54/s in a shear thinning blood viscosity model and thrombosis occurred below this threshold.

In this report, we present a case of an unruptured vertebral artery (VA) aneurysm, for which FAT was performed based on computational fluid dynamics (CFD) results, leading to complete obliteration of the aneurysm without ischemic complications. In addition, we report hemodynamic features associated with intra-aneurysmal thrombus formation.

\section{Case Report}

A 69-year-old female was incidentally found to have an unruptured left VA fusiform aneurysm. Three-dimensional computed tomography (3D-CT) angiography showed the aneurysm at the left V4 segment located distally to the left posterior inferior cerebellar artery (PICA) origin, which had grown over 1 year (Fig. 1A). Intra-aneurysmal thrombosis was not shown on magnetic resonance image. The aneurysm size was $14.8 \mathrm{~mm}$ and posterior communicating arteries on both sides were invisible on 3D-CT angiography. The anterior spinal artery was also not visualized even on digital subtraction angiography (Figs. 1B and 1C). We planned the left VA occlusion at the proximal to the left PICA as the simplest FAT for the aneurysm, and CFD simulations were performed to predict the effects.

\section{CFD Analysis}

CFD analysis was performed as previously reported $^{5)}$ and the method was briefly described as follows. The surface of the arterial lumen was constructed first by using commercially available software packages (Mimics 16.0; Materialise Japan, Yokohama, Japan). The stereolithography (STL) was 
re-meshed to improve the quality of the surface triangles (3-matics 6.0; Materialise Japan, Yokohama, Japan). The computational hybrid meshes were generated with tetrahedral and prism elements (ANSYS ICEM CFD15.0; ANSYS, Inc., Canonsburg, PA, USA). A straight inlet extension was added to the V3 segment of the bilateral VAs to obtain fully developed laminar flow in a control model. On the other hand, a straight inlet extension was added to the V3 segment of the left VA in a FAT model. Thus, bilateral VAs were assumed as inlets in a control model, whereas only the left VA was assumed as an inlet in a FAT model. For the fluid domain, 3D incompressible laminar flow fields were obtained by solving the continuity and Navier-Stokes equations. Numeral modeling was performed using a commercially available CFD package (ANSYS CFX CFD15.0; ANSYS, Inc., Canonsburg, PA, USA). Blood was assumed to be an incompressible Newtonian fluid with blood density of $1056 \mathrm{~kg} / \mathrm{m}^{3}$ and blood viscosity of $0.0035 \mathrm{~Pa} \cdot \mathrm{s}$. Typical VA flow waveform of phase-contrast magnetic resonance imaging was scaled to achieve a physiological WSS. Traction-free boundary conditions were applied to the outlets of posterior cerebral arteries and PICAs. In transient analysis, an initial value was specified with the result of steady state analysis at the first step. The time steps were $0.0001 \mathrm{~s}$, and one pulsatile cycle was taken as output. From the simulated flow fields, we calculated the following hemodynamic parameters: WSS, shear rate, oscillatory shear index (OSI) and aneurysm formation indicator (AFI). WSS indicates the frictional force exerted by the flowing blood tangentially on the dome wall during the cardiac cycle. Shear rate is defined as the rate of increase of blood flow velocity of adjacent streaming layers.

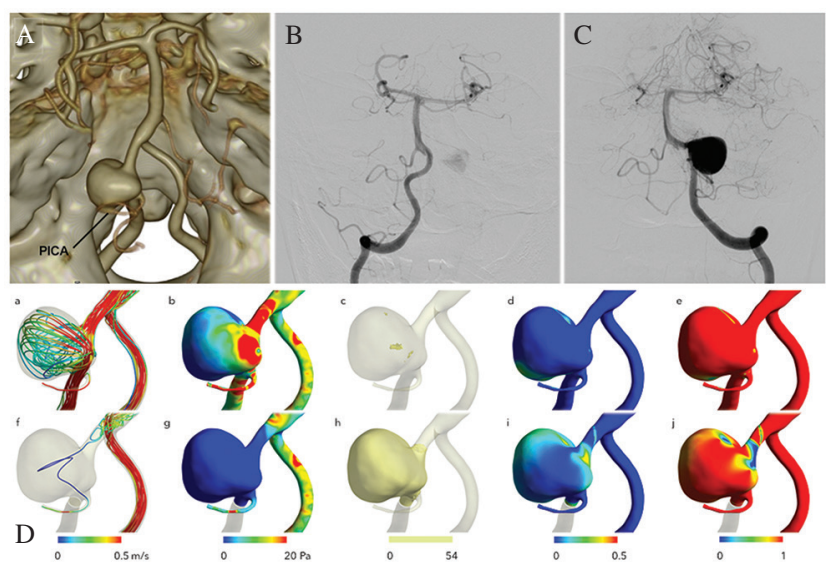

Fig. 1 Preoperative evaluation of aneurysm. (A) Three-dimensional computed tomography angiography shows an aneurysm at the left V4 segment located distally to the left posterior inferior cerebellar artery (PICA) origin. Bilateral posterior communicating arteries are invisible. (B and C) Anterior-posterior views of right (B) and left (C) vertebral angiography show no visualization of the anterior spinal artery. (D) Streamlines $(a, f)$ and distribution of wall shear stress (WSS: $b, g$ ), shear rate $(\mathrm{c}, \mathrm{h}$ ), oscillatory shear index (OSI: $\mathrm{d}, \mathrm{i}$ ), and aneurysm formation indicator (AFI: e, j) in the control (upper) and flow alteration treatment (FAT: lower) models. Computational fluid dynamics simulations show preserved streamlines into the left PICA after the FAT simulation, much lower WSS, shear rate, AFI, and higher OSI at the aneurysm dome in the FAT model compared with the control model.
OSI is defined as the directional changes of WSS during the cardiac cycle. ${ }^{6}$ AFI is defined as an index to detect the flow stagnation zones. ${ }^{7}$ AFI was calculated at the midsystolic deceleration. Other data were time average values over the pulsatile cycle of flow simulation. To evaluate the hemodynamic effects of FAT, hemodynamic parameters at aneurysm dome were compared between the control and FAT models.

Streamlines and distribution of hemodynamic parameters in each model are shown in Fig. 1D. In the control model, WSS and shear rates were 4.59 $\mathrm{Pa}$ and 244 /s, respectively, at the aneurysm dome. In the FAT model, WSS and shear rate were reduced to $0.03 \mathrm{~Pa}$ and 0.76 /s respectively at the aneurysm dome, which were much less than the presumed thresholds necessary to thrombus formation. ${ }^{4)}$ Additionally, WSS and shear rate were $2.93 \mathrm{~Pa}$ and 769 /s, respectively, at the left PICA and streamlines into the left PICA from the distal VA were preserved in the FAT model. The FAT model also had lower AFI and higher OSI than the control model. Taken together, we predicted that the left VA occlusion proximal to the PICA and aneurysm would induce intra-aneurysmal thrombus formation with preservation of the left PICA flow.

\section{FAT and Postoperative Course}

We obtained ethical approval from the ethical committee of our institute and written informed consent from the patient and her relatives prior to the FAT procedure. The operative field was exposed via a lateral suboccipital approach in preparation for the occipital artery (OA) to PICA bypass. The aneurysm precluded the exposure of the left VA distal to the aneurysm. The aneurysmal wall was not thickened and indocyanine green videoangiography clearly visualized the aneurysm (Figs. 2A and 2B). After clipping of the left VA at the
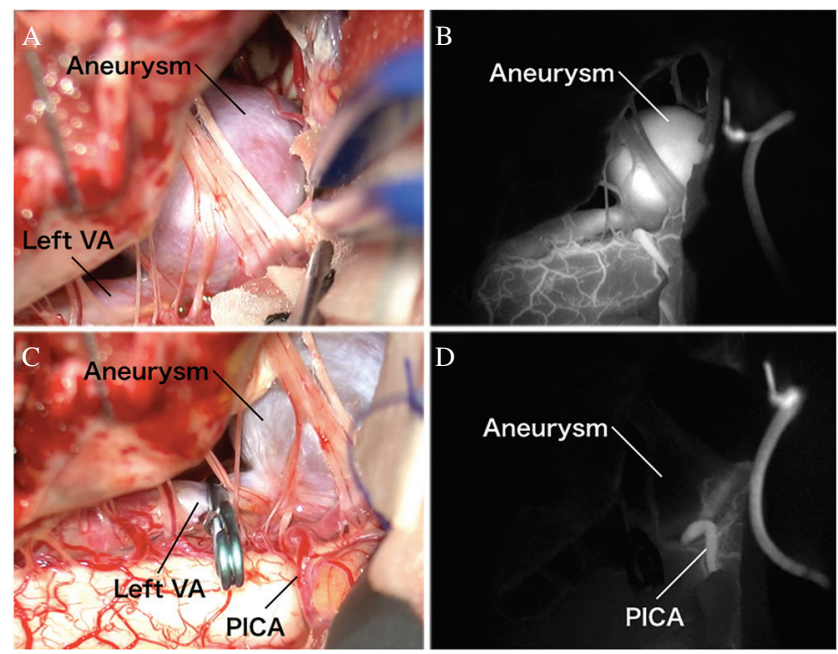

Fig. 2 Intraoperative findings. (A) The operative field is exposed via a left lateral suboccipital approach. The aneurysmal wall is not thickened. (B) Indocyanine green videoangiography clearly shows a vertebral artery (VA) aneurysm. (C) Intraoperative photograph shows clipping of the left VA at the proximal to the left posterior inferior cerebellar artery (PICA) and aneurysm. (D) Indocyanine green videoangiography shows a retrograde blood flow into the left PICA and no filling of the aneurysm dome. 
proximal to the left PICA and the aneurysm, indocyanine green videoangiography was performed again and showed that the aneurysm dome was not filled but a retrograde flow into the left PICA was preserved (Figs. 2C and 2D). Thus, as preoperatively planned, we performed the left VA occlusion at the proximal to the left PICA with neither trapping of the VA nor OA-PICA bypass. The surgical procedure was accomplished successfully without neurological deficits. Neither antiplatelets nor anticoagulants were administered during the perioperative period. The aneurysm was completely obliterated with preservation of the left PICA flow on 3D-CT angiography within 7 days post-surgery (Fig. 3). Three-month follow-up 3D-CT angiography confirmed patency of the left PICA and no aneurysm filling (Fig. 3D). On time-of-flight magnetic resonance images, the reduction of aneurysmal dome was not shown at 2 and 4 months postsurgery, but shown at 2 years post-surgery (Fig. 4).

\section{Discussion}

The goal of treatment for cerebral aneurysms is complete obliteration of the lesion without disturbing cerebral blood flow. Most intracranial aneurysms can be treated with one of two conventional treatments: surgical clipping or endovascular coiling. However, some complicated aneurysms remain impossible to be treated using these methods. The complicated aneurysms have some of the following features: widenecked large or giant aneurysms; dissecting, fusiform or serpentine aneurysms; thrombosed aneurysms; recanalized aneurysms after coil embolization; and perforating vessels
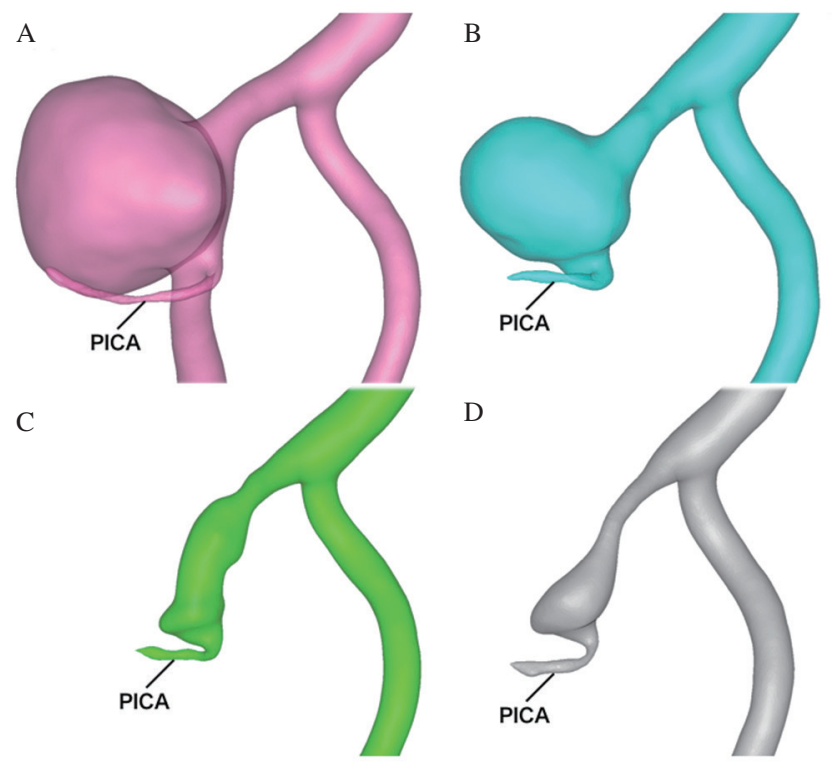

Fig. 3 Chronological changes of the left vertebral artery (VA) aneurysm on three-dimensional computed tomography angiography. (A) Preoperative image shows a large fusiform aneurysm at the left VA just distal to the left posterior inferior cerebellar artery (PICA) origin. (B) At one day post-surgery, the left VA just proximal to the left PICA is invisible and the aneurysm size is obviously decreased with preservation of the left PICA. (C) At 7 days post-surgery, the aneurysm almost disappears, but the left PICA is preserved. (D) At 90 days post-surgery, patency of the left PICA and no aneurysm filling are continued. arising from aneurysmal dome. ${ }^{8)}$ The natural course of complicated aneurysms is unfavorable, but the treatment is also at high risk. ${ }^{9)}$ When the conventional treatments are not feasible, FAT has been performed as a promising alternative. ${ }^{1)}$ However, the efficacy and limitations of FAT have not yet been established. In previous reports, the morbidity of FAT for complicated aneurysms was still more than $15 \%$ including incomplete obliteration of aneurysms and ischemic complications. ${ }^{1)}$

In hemodynamic changes induced by FAT for cerebral aneurysms, both of the flow reduction into an aneurysm and the maintenance of cerebral circulation are very important for successful treatment. Inadequate flow reduction into an aneurysm could not induce complete obliteration of the aneurysm. Incomplete obliteration of aneurysms was associated with postoperative aneurysmal rupture. ${ }^{10)}$ In addition, insufficiency of distal cerebral blood flow may cause ischemic complications. If the hemodynamic changes due to FAT could be quantified and therapeutic effects of FAT could be predicted, FAT would be a more useful and safe therapeutic option for cerebral aneurysms.

WSS and shear rate have been investigated for their potential impact on thrombus formation. ${ }^{2,3)}$ Under normal physiological flow conditions, wall shear rate increases from about $10 / \mathrm{s}$ in veins to about 2000/s in the smallest arteries. The corresponding WSS is typically 0.35 to $70 \mathrm{~Pa}$ in the normal vasculature. ${ }^{11)}$ In a condition of high flow velocity induced by a stenotic lesion, high WSS and high shear rate increase concentration of platelets and activate platelets, which contribute to the rate and extent of intra-arterial thrombus formation. ${ }^{12)}$ On the other hand, thrombus formation is also induced in a condition of flow stagnation. Corbett et al. ${ }^{4)}$ reported that thrombosis occurred below a WSS threshold of $0.41 \mathrm{~Pa}$ or a

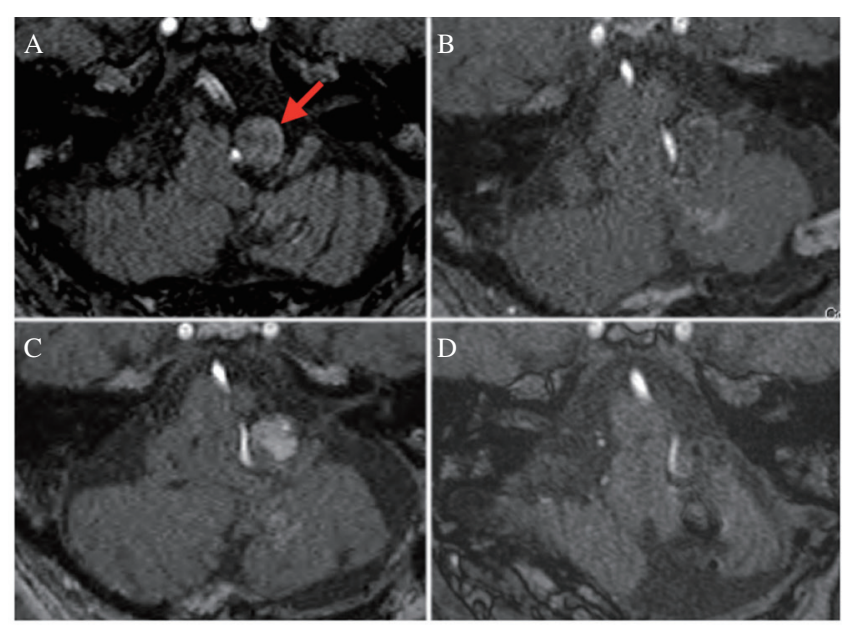

Fig. 4 Chronological changes of the left vertebral artery (VA) aneurysm on three-dimensional time-of-flight magnetic resonance images. (A) Preoperative image shows a large VA aneurysm located on the left side of the brain stem (arrow). (B and C) At 2 (B) and 4 months (C) post-surgery, the patency of the left posterior inferior cerebellar artery (PICA) is continued, while the reduction of aneurysmal dome is not shown. (D) At 2 years post-surgery, the reduction of thrombosed aneurysmal dome is shown with preservation of the left PICA flow. 
shear rate threshold of 54/s in a shear thinning blood viscosity model.

In this case, we performed CFD simulations to determine surgical strategies for an unruptured VA fusiform aneurysm. The FAT model had much lower WSS and shear rate than the presumed thresholds necessary to thrombus formation reported by Corbett et al. ${ }^{4)}$ and the actual surgical treatment achieved the complete obliteration of the aneurysm without ischemic complications. In addition, the FAT model had higher OSI and lower AFI than the control model. High OSI is associated with complex flow, ${ }^{6}$ and low AFI means flow stagnation. ${ }^{7)}$ Our results thus suggest that thrombus formation may be associated with complex flow under a condition of flow stagnation. Meanwhile, the method used in this report might not be applied to simulations of the therapeutic effects of FAT for partially thrombosed VA aneurysms, because presence of intrathrombotic vascular channels and defects of endothelial cells within the vascular lumen might have a significant influence on intra-aneurysmal thrombus formation.

There are several limitations related to CFD analysis in this report. We have assumed in our CFD analysis that the blood was modeled as a Newtonian fluid with blood density of $1056 \mathrm{~kg} / \mathrm{m}^{3}$ and blood viscosity of $0.0035 \mathrm{~Pa} \cdot \mathrm{s}$. In addition, in-flow conditions were not patient-specific but were derived from a representative inlet velocity waveform. Although there are discussions as to what kind of patient-specific data should be adopted for CFD analysis, previous CFD analyses suggested that the most important factor for a realistic representation of the in vivo hemodynamics is the vascular geometry ${ }^{13)}$ The findings in this report are promising, but should be confirmed by large-scale studies.

\section{Conclusion}

Potential usefulness of CFD simulations was reported to determine treatment strategies such as FAT for aneurysms. CFD simulations may become an essential preoperative modality for complicated aneurysms.

\section{Conflicts of Interest Disclosure}

The authors declare that they have no conflicts of interest.

\section{References}

1) Kalani MY, Zabramski JM, Hu YC, Spetzler RF: Extracranialintracranial bypass and vessel occlusion for the treatment of unclippable giant middle cerebral artery aneurysms. Neurosurgery 72: 428435; discussion 435-436, 2013

2) Baumgartner HR: The role of blood flow in platelet adhesion, fibrin deposition, and formation of mural thrombi. Microvasc Res 5: 167-179, 1973

3) Sakariassen KS, Turitto VT, Baumgartner HR: Recollections of the development of flow devices for studying mechanisms of hemostasis and thrombosis in flowing whole blood. J Thromb Haemost 2: 16811690,2004

4) Corbett SC, Ajdari A, Coskun AU, N-Hashemi H: In vitro and computational thrombosis on artificial surfaces with shear stress. Artif Organs 34: 561-569, 2010

5) Miura Y, Ishida F, Umeda Y, et al.: Low wall shear stress is independently associated with the rupture status of middle cerebral artery aneurysms. Stroke 44: 519-521, 2013

6) Taylor CA, Hughes TJ, Zarins CK: Finite element modeling of threedimensional pulsatile flow in the abdominal aorta: relevance to atherosclerosis. Ann Biomed Eng 26: 975-987, 1998

7) Mantha A, Karmonik C, Benndorf G, Strother C, Metcalfe R: Hemodynamics in a cerebral artery before and after the formation of an aneurysm. AJNR Am J Neuroradiol 27: 1113-1118, 2006

8) Drake CG, Peerless SJ: Giant fusiform intracranial aneurysms: review of 120 patients treated surgically from 1965 to 1992. J Neurosurg 87: 141-162, 1997

9) Drake CG, Peerless SJ, Ferguson GG: Hunterian proximal arterial occlusion for giant aneurysms of the carotid circulation. J Neurosurg 81: 656-665, 1994

10) Takai N, Ezuka I, Sorimachi T, Kumagai T, Sano K: Vertebral artery dissecting aneurysm rebleeding after proximal occlusion-case report. Neurol Med Chir (Tokyo) 33: 765-768, 1993

11) Strony J, Beaudoin A, Brands D, Adelman B: Analysis of shear stress and hemodynamic factors in a model of coronary artery stenosis and thrombosis. Am J Physiol 265: H1787-H1796, 1993

12) Roux SP, Sakariassen KS, Turitto VT, Baumgartner HR: Effect of aspirin and epinephrine on experimentally induced thrombogenesis in dogs. A parallelism between in vivo and ex vivo thrombosis models. Arterioscler Thromb 11: 1182-1191, 1991

13) Cebral JR, Pergolizzi RS, Putman CM: Computational fluid dynamics modeling of intracranial aneurysms: qualitative comparison with cerebral angiography. Acad Radiol 14: 804-813, 2007

Corresponding author:

Yoichi Miura, MD, PhD, Department of Neurosurgery, Suzuka Kaisei Hospital, 112-1 Kou, Suzuka, Mie 513-8505, Japan.

$\triangle$ yoimiu@hotmail.com 